\title{
PREFACE
}

The physics of Wolf-Rayet stars has major highlights: they are the most advanced observed stage of massive star evolution, progenitors of supernovae and sources of cosmic rays. They are the result of massive star evaporation and provide nucleosynthesis tests. Just as WR stars appear to represent a particular phase in the evolution of massive stars, WR galaxies represent a stage in the evolution of the dominant starburst stellar population in dwarf irregular galaxies or $\mathrm{H}$ II galaxies. During the last decade the major role of starbursts in the evolution of the universe has become evident. In recent years we have seen avalanches of new observational results in the field of WR stars and WR galaxies E.g., new observations in the centre of our own Galaxy reveal a large number of hot luminous helium-rich stars of WR-type and Of/WN-type, whose winds may have a profound influence on the evolution of the Galactic Centre. The spectroscopic WR phenomenon allows massive starbursts to be observed out to huge distances, and thus to be studied at greater variety of metallicities and ages of the universe. While these observational developments are taking place on both the extragalactic and stellar fronts, also on the theory and modelling side there have been, and will continue to be, important developments in atmosphere modelling techniques, galaxy/starburst spectral synthesis and stellar evolution modelling. In the 1990-ies already over 2000 papers on WR stars and WR galaxies have been published (see, e.g., the WR bibliography section of the Hot Star Newsletter on http://www.star.ucl.ac.uk/ hsn/index.html).

The first ideas for this Symposium were generated at informal gatherings during IAU Symposium No. 163 on Wolf-Rayet Stars: Binaries Colliding Winds, Evolution, on Elba in May 1994. At the time it was felt that in a subsequent IAU symposium on Wolf-Rayet stars, aspects of starbursts should receive specific attention. A candidate Scientific Organizing Committee was invited in Spring 1995 , and extensive communication among its members subsequently established the scientific programme for the symposium. The basic structure agreed upon was: (1) basic observational properties of WR stars and other hot massive stars; (2) models for single star evolution of massive stars: winds + atmospheres + interior; (3) interaction of WR stars and other hot massive stars with their environment: colliding winds and ring nebulae; (4) WR stars and other hot massive stars in the Galactic Center and in Local Group giant H II regions; (5) WR stars and other hot massive stars in starburst galaxies: the case of WR galaxies; and (6) starbursts in normal and active galaxies: their effects on the ISM and chemical evolution.

Following endorsement and sponsoring by IAU Commissions and Divisions and subsequent approval by the IAU Executive Committee in August 1997, the SOC and LOC were formalized, speakers were invited, and the community at large informed. Thanks to the overwhelmingly positive response, the scientific programme comprised 54 invited oral presentations and 143 poster papers, entertaining 186 astronomers from 24 countries.

Tradition prescribes that IAU symposia on hot massive stars take place in high-luminosity beach resorts, and after IAU Symposia in Buenos Aires (No. 49), Qualicum Beach (No. 83), Cozumel (No. 99), Porto Heli (No. 116), Bali (No. 143) and Elba (No.163), Puerto Vallarta proved to be an excellent location in this 
series. A first report of the symposium has been published by SOC-member Peter S. Conti (1999, PASP 111, 251).

The Local Organizing Committee, under the expert guidance of its chairman, did an excellent job, not only in hosting us at an extremely efficient and pleasant venue, but also in distributing the financial support from the IAU, the UNAM, and the local sponsors: CLAFM and the University of Guanajuato.

Thanks to the cooperation of the authors, most manuscripts arrived in time and in good shape, thus alleviating the task of the editors. All but the poster papers have been thoroughly refereed by members of the SOC. The discussion registration was in the able hands of Luis Corral (IAC), and decipherment of the discussion sheets has been performed skilfully by Ms. Sandra Blom (SRON).

Four years ago we departed from Elba. We have been wandering about in the vessel of science on the high seas of thought, to make landfall in Puerto Vallarta, to refuel and to take on fresh supplies, i.e.,, for a week of exchange and stimulation. And while the waves of the Pacific Ocean were crashing constantly on the beaches of Puerto Vallarta, waves of information on new observations and theories came crashing continuously on to us participants in the auditorium. Many directions for future research have been straightened out to various degrees, and all of us have found motivation and incentives to carry on. May these Proceedings be a compass to guide us in the ocean of research on massive stars in general and on Wolf-Rayet phenomena in particular, till a beach resort for a next symposium on Wolf-Rayet phenomena looms at the horizon.

\section{The editors:}

Karel A. van der Hucht, Gloria Koenigsberger, and Philippe R.J. Eenens Utrecht, Mexico City, Guanajuato, May 7, 1999

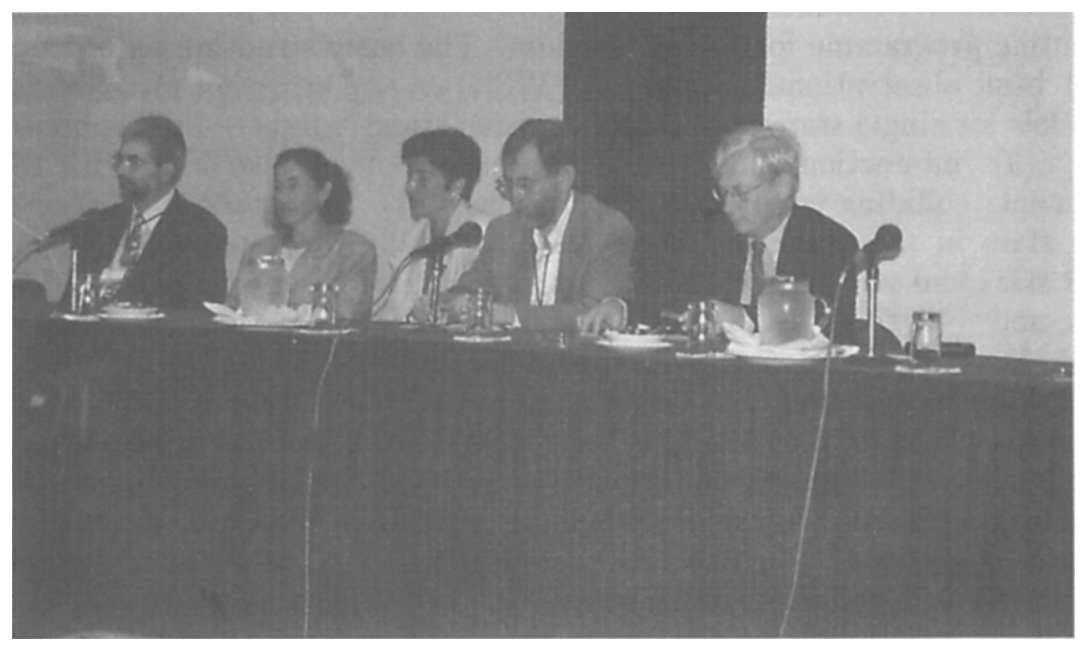

